\title{
Brentuximab vedotin-containing escalated BEACOPP variants for newly diagnosed advanced-stage classical Hodgkin lymphoma: follow-up analysis of a randomized phase II study from the German Hodgkin Study Group
}

Carla Damaschin $^{1,2}$, Helen Goergen ${ }^{1,2}$, Stefanie Kreissl ${ }^{1,2}$, Annette Plütschow $^{1,2}$, Frank Breywisch ${ }^{3}$, Stephan Mathas (DD ${ }^{4}$, Julia Meissner ${ }^{5}$, Martin Sökler ${ }^{6}$, Max S. Topp ${ }^{7}$, Vladan Vucinic ${ }^{8}$, Andreas Zimmermann $^{9}$, Bastian von Tresckow ${ }^{2,10}$, Michael Fuchs ${ }^{1,2}$, Andreas Engert ${ }^{1,2}$, Peter Borchmann ${ }^{1,2}$ and Dennis A. Eichenauer (iD ${ }^{1,2}$

(c) The Author(s) 2021

Leukemia (2022) 36:580-582; https://doi.org/10.1038/s41375-021-01386-z

Patients with advanced-stage Hodgkin lymphoma $(\mathrm{HL})$ receiving intensive treatment with escalated BEACOPP (bleomycin, etoposide, doxorubicin, cyclophosphamide, vincristine, procarbazine, prednisone) (eBEACOPP) have excellent outcomes [1, 2]. However, late effects such as second primary malignancies (SPM) and infertility represent ongoing concerns $[3,4]$. Novel approaches using an eBEACOPP backbone, therefore, aim at reducing toxicity without compromising efficacy. The BrECAPP (brentuximab vedotin, etoposide, cyclophosphamide, doxorubicin, procarbazine, prednisone) and BrECADD (brentuximab vedotin, etoposide, cyclophosphamide, doxorubicin, dacarbazine, dexamethasone) protocols were investigated in a randomized phase II study including patients with newly diagnosed advanced-stage classical $\mathrm{HL}$ ( $\mathrm{CHL}$ ). Both regimens combine eBEACOPP-based chemotherapy with the CD30-directed antibody-drug conjugate brentuximab vedotin (BV). Response rates were similar to standard eBEACOPP and especially the BrECADD protocol was associated with reduced acute toxicity [5].

The major objectives of the present follow-up analysis of the study were progression-free survival (PFS) and overall survival (OS) at 3 years. Patterns of $\mathrm{CHL}$ recurrence, the occurrence of SPM, and causes of death were also evaluated. PFS and OS were analyzed using the Kaplan-Meier method. PFS was defined as the time between randomization and progression or relapse of $\mathrm{cHL}$ or death from any cause and was censored at the date of last information on the disease status. OS was defined as the time between randomization and death from any cause and was censored at the date of last information for surviving patients.
Characteristics of $\mathrm{cHL}$ recurrence, SPM, and causes of death were analyzed descriptively. Study design, inclusion criteria, endpoints, procedures, and treatment have been published elsewhere [5]. The study was conducted in accordance with the Declaration of Helsinki and was approved by the review boards of the participating sites. The study was registered at www.clinicaltrials. gov as \#NCT01569204.

A total of 104 patients with advanced-stage $\mathrm{cHL}$ aged 18-60 years were enrolled at 20 sites in Germany. Baseline characteristics have been reported previously. In brief, 63 patients (61\%) were male, the median age was 29 years (range: 18-60 years), and 86 patients (82\%) presented with stage III/IV disease [5]. Baseline characteristics were thus consistent with the randomized German Hodgkin Study Group (GHSG) HD15 and HD18 studies for advanced-stage $\mathrm{HL}[1,2]$. Three patients were not included in the outcome analyses since they did not receive study treatment $(n=2)$ or terminated study treatment after the advanced-stage disease had been disconfirmed $(n=1)$. Hence, the present analysis included 101 patients who had been treated with 6 cycles of BrECAPP $(n=49)$ or BrECADD $(n=52) ; 13$ patients (BrECAPP: 7 patients; BrECADD: 6 patients) with residual lymphoma $\geq 2.5 \mathrm{~cm}$ and a positive positron emission tomography (PET) at the end of systemic therapy had an indication for consolidation radiotherapy [5].

After a median observation time of 34 months (interquartile range: $28.7-39.5$ months), the 3-year PFS estimates were $90.2 \%$ (95\%-Cl: 80.9-99.5\%) with BrECAPP and $89.7 \%$ (95\%-Cl: 81.0-98.3\%) with BrECADD (Fig. 1A, Table 1). In the randomized

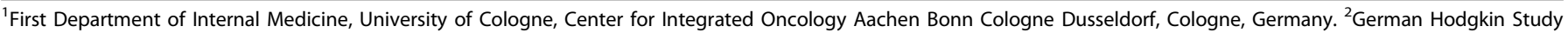

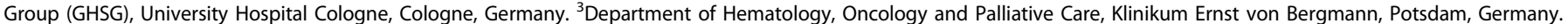

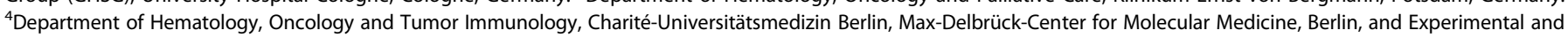
Clinical Research Center (ECRC), Berlin, Germany. ${ }^{5}$ Fifth Department of Internal Medicine, University Hospital Heidelberg, Heidelberg, Germany. ${ }^{6}$ Second Department of Internal

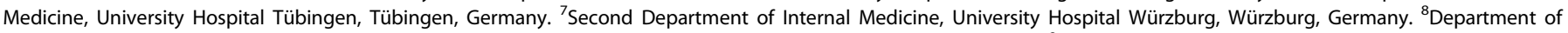

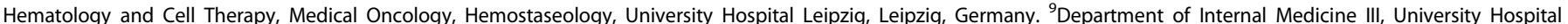

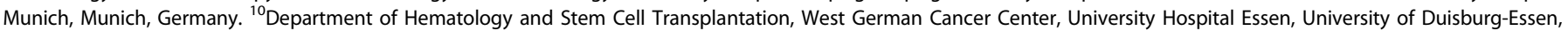
Essen, Germany. ${ }^{凶}$ email: dennis.eichenauer@uk-koeln.de
} 
A

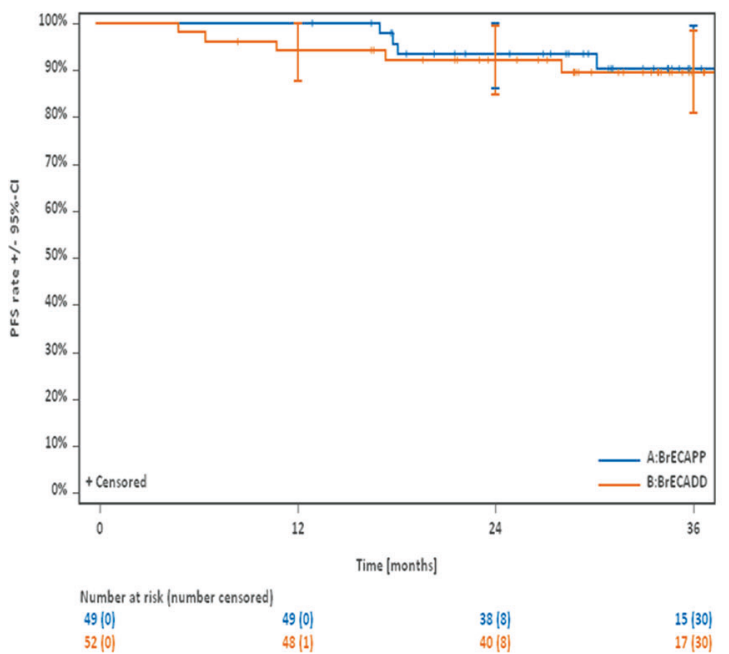

B

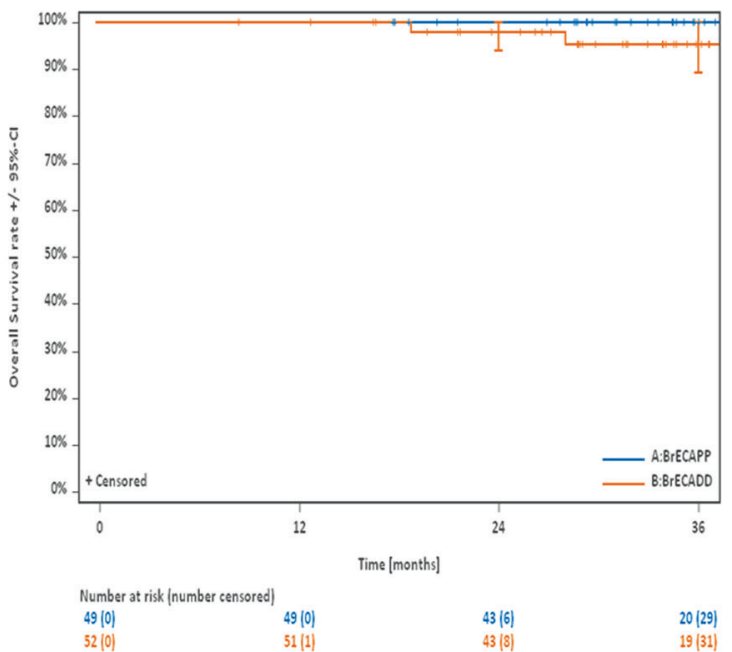

Fig. 1 Outcomes of patients treated with BrECAPP and BrECADD. A) Progression-free survival after treatment with BrECAPP and BrECADD; B) Overall survival after treatment with BrECAPP and BrECADD.

Table 1. Outcomes and events after BrECAPP and BrECADD treatment.

\begin{tabular}{|c|c|c|}
\hline & $\begin{array}{l}6 \times \text { BrECAPP } \\
(N=49)\end{array}$ & $\begin{array}{l}6 \times \text { BrECADD } \\
(N=52)\end{array}$ \\
\hline \multicolumn{3}{|l|}{ Observation time } \\
\hline Observation time for disease status, median & 31 months & 34 months \\
\hline Observation time for survival status, median & 35 months & 34 months \\
\hline \multicolumn{3}{|l|}{ 3-year survival estimates } \\
\hline Progression-free survival & $\begin{array}{l}90.2 \% \\
(80.9-99.5 \%)\end{array}$ & $\begin{array}{l}89.7 \% \\
(81.0-98.3 \%)\end{array}$ \\
\hline Overall survival & $100 \%$ & $\begin{array}{l}95.4 \% \\
(89.2-100 \%)\end{array}$ \\
\hline \multicolumn{3}{|l|}{ Hodgkin lymphoma events $^{\mathrm{a}}$} \\
\hline Any Hodgkin lymphoma event & $(8 \%)$ & (8\%) \\
\hline Progression $^{b}$ & 0 & $(6 \%)$ \\
\hline Relapse $^{c}$ & $(8 \%)$ & $(2 \%)$ \\
\hline \multicolumn{3}{|l|}{ Number of Hodgkin lymphoma events } \\
\hline 1 & $(8 \%)$ & $(4 \%)$ \\
\hline 2 & 0 & (4\%) \\
\hline \multicolumn{3}{|l|}{ Second-line treatment } \\
\hline $\begin{array}{l}\text { High-dose chemotherapy and autologous stem } \\
\text { cell transplantation }\end{array}$ & (8\%) & (8\%) \\
\hline \multicolumn{3}{|l|}{ Causes of death } \\
\hline Any event & 0 & (4\%) \\
\hline Hodgkin lymphoma & 0 & $1 \quad(2 \%)$ \\
\hline Accident & 0 & (2\%) \\
\hline \multicolumn{3}{|l|}{ Second primary malignancies } \\
\hline Any event & 0 & 0 \\
\hline
\end{tabular}

Data are median, \% (95\%-Cl), or $n(\%)$. Only patients eligible for efficacy analyses are shown; 3 of 104 patients were excluded because they did not receive any study treatment $(n=2)$ or terminated treatment due to disconfirmation of advanced-stage disease $(n=1)$.

BrECAPP brentuximab vedotin, etoposide, cyclophosphamide, doxorubicin, procarbazine, prednisone, BrECADD brentuximab vedotin, etoposide, cyclophosphamide, doxorubicin, dacarbazine, dexamethasone.

a Defined as biopsy-proven disease progression or relapse of $\mathrm{CHL}$.

${ }^{b}$ Defined as the occurrence of new lesions during treatment or at least 1 known lesion that increased by more than $25 \%$ in diameter during treatment or within 3 months after the end of systemic study treatment. cDefined as the appearance of new lesions or the reappearance of initial lesions at least 3 months after the end of systemic study treatment.
ECHELON-1 study for newly diagnosed stage III/IV cHL, the 3-year PFS among patients aged younger than 60 years who had treatment with the BV-containing A-AVD (BV, doxorubicin, vinblastine, dacarbazine) regimen was $84.9 \% \quad(95 \%-\mathrm{Cl}$ : 81.6-87.7\%) [6]. Disease control with BrECAPP and BrECADD was thus at least similar to and possibly better than with A-AVD.

Eight patients treated in the present study experienced primary disease progression $(n=3)$ or relapse of $\mathrm{cHL}(n=5)$. Sites of initial bulky disease were involved in 6 cases. The median time from the end of BrECAPP or BrECADD therapy to disease progression or relapse was 13 months (range: 1-26 months). Second-line treatment consisted of high-dose chemotherapy and autologous stem cell transplantation in all cases. No study participant developed an SPM (Table 1). This is notable since older analyses had indicated an increased risk especially for the development of therapy-related acute myeloid leukemia and myelodysplastic syndrome (t-AML/ MDS) after 8 cycles of eBEACOPP $[3,7,8]$. However, the current GHSG standard of care for advanced-stage HL consisting of 4 or 6 cycles of eBEACOPP stratified by PET after 2 cycles of chemotherapy appears to be associated with a significantly lower risk. The t-AML/ MDS incidence among patients who had received 4 or 6 cycles of eBEACOPP within the HD15 and HD18 studies was lower than $1 \%$ $[1,2]$. Unlike second hematologic malignancies that usually occur within the first years after $\mathrm{HL}$ treatment, the risk for the development of second solid tumors after BrECAPP and BrECADD cannot be estimated yet $[7,9,10]$. Longer follow-up is required since second solid tumors are typically diagnosed many years after $\mathrm{HL}$ treatment and the risk for the occurrence of such malignancies seems to be elevated for decades [11, 12].

Two patients who had treatment in the present study died during observation. Deaths occurred 15 and 24 months after the end of systemic study treatment. One of the deaths was due to $\mathrm{cHL}$ (Table 1). A low rate of $\mathrm{HL}$-related deaths was also observed in other studies comprising patients with advanced-stage $\mathrm{HL}$, e.g., the HD15 and HD18 studies using eBEACOPP and the RATHL study using ABVD [1, 2, 13].

The 3-year OS estimates were 100\% with BrECAPP and $95.4 \%$ (95\% Cl: 89.2-100\%) with BrECADD (Fig. 1B, Table 1). In the ECHELON-1 study, the 2-year OS among patients treated with A-AVD was $96.6 \%$ [14].

The present analysis has some limitations. Given the mostly young age at diagnosis and the high cure rate, quality of life (QoL) 
aspects and fertility issues play an important role in the management and the choice of treatment in $\mathrm{HL}$ patients [4, 15]. However, valid analyses on QoL and fertility in individuals who had received BrECAPP or BrECADD could not be performed due to the inability to obtain sufficient data.

Taken together, the present update analysis of a randomized phase II study investigating the BV-containing eBEACOPP variants BrECAPP and BrECADD in the first-line treatment of advancedstage $\mathrm{CHL}$ confirms the safety and efficacy of these protocols. The BrECADD regimen had been chosen to challenge eBEACOPP in the randomized GHSG HD21 study (NCT02661503) that recently finished recruitment for the cohort of patients aged 60 years or younger. Results of this trial are pending. Results were in part presented at the EHA 2021 Virtual Congress, June 9 to 17, 2021.

\section{REFERENCES}

1. Engert A, Haverkamp $H$, Kobe $C$, Markova J, Renner $C, H o$ A et al. Reducedintensity chemotherapy and PET-guided radiotherapy in patients with advanced stage Hodgkin's lymphoma (HD15 trial): a randomised, open-label, phase 3 noninferiority trial. Lancet. 2012;379:1791-9.

2. Borchmann P, Goergen H, Kobe C, Lohri A, Greil R, Eichenauer DA, et al. PETguided treatment in patients with advanced-stage Hodgkin's lymphoma (HD18): final results of an open-label, international, randomised phase 3 trial by the German Hodgkin Study Group. Lancet. 2017. https://doi.org/10.1016/S0140-6736 (17)32134-7.

3. Eichenauer DA, Becker I, Monsef I, Chadwick N, de Sanctis V, Federico M, et al. Secondary malignant neoplasms, progression-free survival and overall survival in patients treated for Hodgkin lymphoma: a systematic review and meta-analysis of randomized clinical trials. Haematologica. 2017;102:1748-57.

4. Behringer $\mathrm{K}$, Mueller $\mathrm{H}$, Goergen $\mathrm{H}$, Thielen $\mathrm{I}$, Eibl AD, Stumpf $\mathrm{V}$, et al. Gonadal function and fertility in survivors after Hodgkin lymphoma treatment within the German Hodgkin Study Group HD13 to HD15 trials. J Clin Oncol. 2013;31:231-9.

5. Eichenauer DA, Plutschow A, Kreissl S, Sokler M, Hellmuth JC, Meissner J, et al. Incorporation of brentuximab vedotin into first-line treatment of advanced classical Hodgkin's lymphoma: final analysis of a phase 2 randomised trial by the German Hodgkin Study Group. Lancet Oncol. 2017;18:1680-7.

6. Straus DJ, Długosz-Danecka M, Alekseev S, Illés Á, Picardi M, Lech-Maranda E, et al. Brentuximab vedotin with chemotherapy for stage III/IV classical Hodgkin lymphoma: 3-year update of the ECHELON-1 study. Blood 2020;135. https://doi. org/10.1182/blood.2019003127.

7. Eichenauer DA, Thielen I, Haverkamp H, Franklin J, Behringer $\mathrm{K}$, Halbsguth T, et al. Therapy-related acute myeloid leukemia and myelodysplastic syndromes in patients with Hodgkin lymphoma: a report from the German Hodgkin Study Group. Blood. 2014;123:1658-64.

8. von Tresckow B, Kreissl S, Goergen H, Brockelmann PJ, Pabst T, Fridrik M, et al. Intensive treatment strategies in advanced-stage Hodgkin's lymphoma (HD9 and HD12): analysis of long-term survival in two randomised trials. Lancet Haematol. 2018;5:e462-e473.

9. Koontz MZ, Horning SJ, Balise R, Greenberg PL, Rosenberg SA, Hoppe RT, et al. Risk of therapy-related secondary leukemia in Hodgkin lymphoma: the Stanford University experience over three generations of clinical trials. J Clin Oncol. 2013;31:592-8.

10. Eichenauer DA, Müller $H$, Elger L, Goergen $H$, Fuchs $M$, Kreissl $S$, et al. NonHodgkin lymphoma after treatment for classical Hodgkin lymphoma: a report from the German Hodgkin Study Group. Br J Haematol. 2021;193. https://doi.org/ 10.1111/bjh.17327.

11. Swerdlow AJ, Higgins CD, Smith P, Cunningham D, Hancock BW, Horwich A, et al. Second cancer risk after chemotherapy for Hodgkin's lymphoma: a collaborative British cohort study. J Clin Oncol. 2011;29:4096-104.
12. Schaapveld $M$, Aleman $B M$, van Eggermond $A M$, Janus $C P$, Krol $A D$, van der Maazen RW, et al. Second cancer risk up to 40 years after treatment for Hodgkin's Lymphoma. N Engl J Med. 2015;373:2499-511.

13. Johnson P, Federico M, Kirkwood A, Fossa A, Berkahn L, Carella A, et al. Adapted treatment guided by interim PET-CT scan in advanced Hodgkin's Lymphoma. $\mathrm{N}$ Engl J Med. 2016;374:2419-29.

14. Connors JM, Jurczak W, Straus DJ, Ansell SM, Kim WS, Gallamini A, et al. Brentuximab vedotin with chemotherapy for stage III or IV Hodgkin's Lymphoma. N Engl J Med. 2018;378:331-44.

15. Kreiss S, Mueller H, Goergen H, Mayer A, Brillant C, Behringer K, et al. Cancerrelated fatigue in patients with and survivors of Hodgkin's lymphoma: a longitudinal study of the German Hodgkin Study Group. Lancet Oncol. 2016;17:1453-62.

\section{AUTHOR CONTRIBUTIONS}

$C D, S K, F B, S M, J M, M S, M S T, V V, A Z, B V T, M F, A E, P B$, and DAE provided study material or patients. $C D, H G, A P$, and $D A E$ analyzed data. $C D, H G$, and $D A E$ wrote the manuscript. All authors approved the final manuscript.

\section{FUNDING}

Open Access funding enabled and organized by Projekt DEAL.

\section{COMPETING INTERESTS}

BVT is an advisor or consultant for Amgen, BMS/Celgene, Novartis, Pentixafarm, Pfizer, Takeda, Merck Sharp \& Dohme, and Gilead Kite; has received honoraria from Novartis, Roche Pharma AG, Takeda, and Merck Sharp \& Dohme; reports research funding from Novartis (Inst), Merck Sharp \& Dohme (Inst), and Takeda (Inst); and reports travel support from AbbVie, AstraZeneca, KiteGilead, Merck Sharp \& Dohme, Takeda, and Novartis. AZ has received honoraria from Takeda; and reports travel grants from Takeda. All other authors report no potential conflicts of interest.

\section{ADDITIONAL INFORMATION}

Correspondence and requests for materials should be addressed to D.A.E.

Reprints and permission information is available at http://www.nature.com/ reprints

Publisher's note Springer Nature remains neutral with regard to jurisdictional claims in published maps and institutional affiliations.

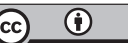

Open Access This article is licensed under a Creative Commons Attribution 4.0 International License, which permits use, sharing, adaptation, distribution and reproduction in any medium or format, as long as you give appropriate credit to the original author(s) and the source, provide a link to the Creative Commons license, and indicate if changes were made. The images or other third party material in this article are included in the article's Creative Commons license, unless indicated otherwise in a credit line to the material. If material is not included in the article's Creative Commons license and your intended use is not permitted by statutory regulation or exceeds the permitted use, you will need to obtain permission directly from the copyright holder. To view a copy of this license, visit http://creativecommons. org/licenses/by/4.0/.

(c) The Author(s) 2021 